\title{
SFG Realization of Wavelet Filter Using Switched-Current Circuits
}

\author{
Wenshan Zhao, Yigang He, and Yichuang Sun
}

\begin{abstract}
A signal flow graph method for analogue implementation of wavelet transform using switched-current circuits is proposed in which the wavelet transform is synthesized by a bank of switched-current bandpass filters whose impulse responses are the mother wavelet and its dilations. To facilitate the implementation of arbitrary wavelet function, the proposed approach employs the signal flow graph methodology to design the wavelet filter. The first derivative of Gaussian wavelet is used as an example in this paper to illustrate design details. Simulation results show the feasibility of the proposed method.
\end{abstract}

Index Terms - Wavelet transform, SFG, Switched-current

\section{INTRODUCTION}

Wavelet transform (WT) has found a wide range of applications in signal processing due to its time-frequency localization characteristics [1]. Because of the heavy computational burden, however, software implementations of wavelet transforms cannot be used in real-time signal processing. Over the past few years, hardware implementations have been investigated [2,3], mostly concentrating on digital circuit implementations. Although successful in many aspects, these designs suffer from the effect of large power dissipation associated with the required analogue-digital converter. To alleviate the difficulties associated with such digital designs, research on analogue circuit implementations has attracted much attention [4-8]. Typically, [7] presents an approach to implement wavelet transforms using switched-current (SI) circuits, in which the wavelet transform is synthesized by a bank of SI filters whose impulse responses are the basic wavelet function and its dilations (i.e. wavelet filter). This method resolves the problem of incompatibility with the digital VLSI technology when implementing with switched-capacitor

\footnotetext{
${ }^{1}$ This work was supported by the National Natural Science Foundation of China under Grant No.60876022, and Doctoral Special Fund of Ministry of Education of China under Grant No. 20060532002, High-Tech Research and Development Program of China under Grant No. 2006AA 04A 104.

Wenshan Zhao and Yigang $\mathrm{He}$ are with the College of Electrical \& Information Engineering, Hunan University, Changsha, Hunan 410082, China (e-mail: \{shmilyzhyao, hyghnu \}@yahoo.com.cn).

Wenshan Zhao is also and Yichuang Sun is with the School of ECEE, University of Hertfordshire, Hatfield, Herts AL10 9AB, UK (e-mail: Y.Sun@herts.ac.uk).
}

(SC) filters. Also, it facilitates the circuit design greatly since the various dilations of SI wavelet filters can be implemented by controlling the sampling frequency precisely and easily.

As of now, the prevalent methods for analogue implementation of WT mainly involves the rational function approximation of wavelet bases and the design of bandpass filters whose impulse responses are the approximated wavelet bases. In [7], the wavelet base is synthesized with a rational function obtained by the Padé approximation, and then realized by a cascade connection of SI filters. Although the feasibility is verified in this paper, this method still has some limitations as below: Firstly, the zeros and poles of the approximation network are arbitrary and unpredictable. In many cases, the approximation rational function cannot be factorized into a multiplication of several rational fractions, which means it is impossible to be realized by cascade filters. Secondly, although the cascade connection performs relatively well, their passband sensitivities tend to become too large for many applications, especially as the filter order increases [9].

To overcome the shortcomings appeared in [7], this paper proposes a method that employs the powerful signal flow graph (SFG) methodology to implement arbitrary approximation rational functions. For brevity, here we use the approximation rational function of the first derivative of Gaussian wavelet given in [7] as an example to illustrate the design procedure, in which the SI bilinear mapping lossless integrator is used as the building block. The feasibility of the proposed method is confirmed by simulation.

\section{Wavelet Design Procedure}

Assuming $\psi(t)$ is the mother wavelet (or wavelet base), the wavelet transform of the signal $x(\mathrm{t})$ at the scale $a$ and timeshift $b$ can be defined by convolving $x(\mathrm{t})$ with a dilated wavelet [1],

$$
W T_{x}(a, b)=a^{-1} \int x(t) \psi\left(\frac{b-t}{a}\right) d t
$$

The coefficient, $1 / a$, maintains the amplitude of frequency response of $\psi_{a, b}=a^{-1} \psi\left(\frac{b-t}{a}\right)$ across different scales. Equivalently, (1) can be expressed in terms of the Fourier transforms of the signal and wavelet

978-1-4244-3870-9/09/\$25.00 C2009 IEEE 


$$
W T_{\mathrm{x}}(a, b)=[1 /(2 \pi)] \int X(\omega) \Psi^{*}(a \omega) \exp (j \omega b) d \omega
$$

Due to the characteristic of satisfying the admissibility criteria [1], the wavelets are inherently bandpass filters in the Fourier domain, defined as wavelet filters. Hence, the WT can be carried out simply by using a bank of bandpass filters whose impulse responses are the mother wavelet and its dilated versions.

\section{Signal Flow Graphs For ARbitrary Transfer FUNCTIONS}

The general transfer function with arbitrary finite transmission zeros can be defined as

$$
H(s)=\frac{a_{n} s^{n}+a_{n-1} s^{n-1}+\cdots+a_{2} s^{2}+a_{1} s^{1}+a_{0}}{b_{n} s^{n}+b_{n-1} s^{n-1}+\cdots+b_{2} s^{2}+b_{1} s^{1}+b_{0}}
$$

Based on Mason's Gain Formula, the direct signal flow graph for a current-mode circuit which has the transfer function as (3) can be realized by Fig.1 (a) [10]. For the convenience of practical realization, one can replace the integrator $(1 / \mathrm{s})$ by the general integrator $(1 / \mathrm{s} \sigma)$. Meanwhile, in order to give the same transfer function, the corresponding modifications of the feedback and feedforward coefficients should be made accordingly. After scaling, the modified SFG of (3) is shown in Fig. 1 (b).

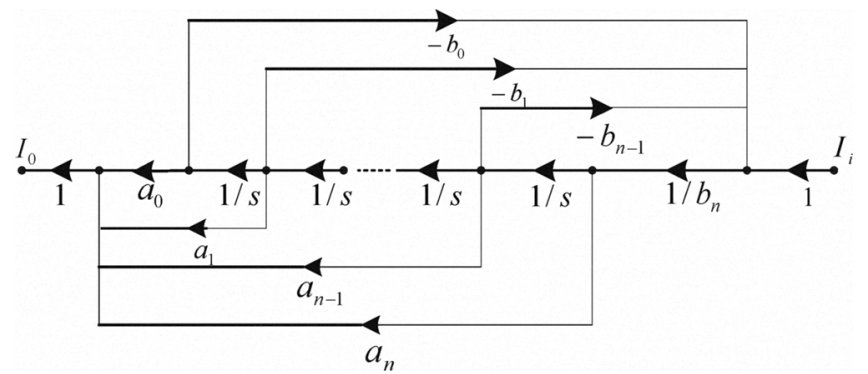

(a)

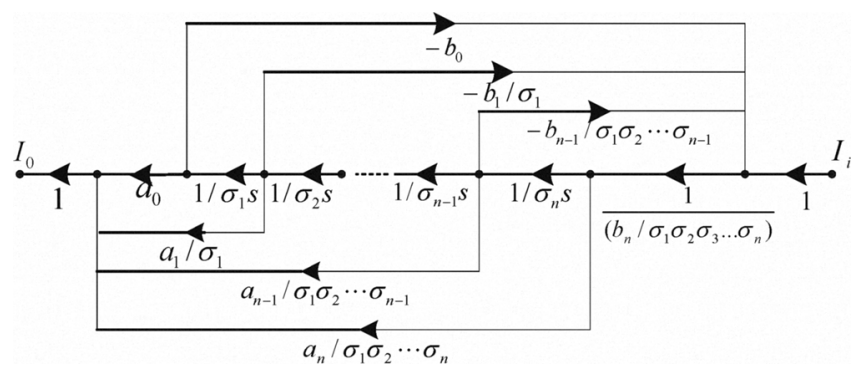

(b)

Fig.1. (a) Direct form and (b) Modified signal flow graph for currentmode

\section{WAVELET FILTER DESIGN}

\section{A. Switched-Current Building Blocks}

As seen from Fig.1, the basic building cell of the filter architecture is the general integrator $1 / \mathrm{s} \sigma$. In this paper, the bilinear mapping lossless integrator based on the second- generation SI circuit as shown in Fig. 2 is used as the integrator block [11]. Its transfer functions in z-domain and sdomain are defined as (4) and (5), respectively.

$$
\begin{aligned}
& H(z)=\alpha \frac{z+1}{z-1} \\
& H(s)=1 / \sigma s
\end{aligned}
$$

Using bilinear transform, $s=\frac{2}{T} \frac{(z-1)}{(z+1)}$, the parameter value in Fig. 2 can be calculated by the coefficient matching as

$$
\alpha=T / 2 \sigma
$$

in which, $\mathrm{T}$ is the sampling period of the SI circuit.

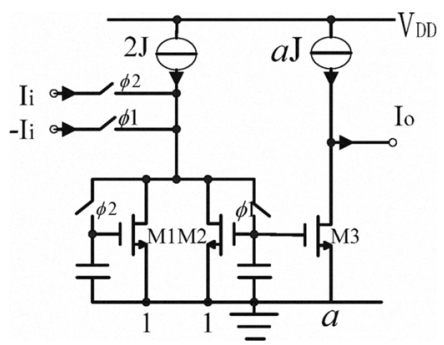

Fig.2. Bilinear mapping lossless integrator

\section{B. Filter Architecture and Synthesis}

The normalized approximation function for the first derivative of Gaussian wavelet proposed in [7] is given as:

$$
H(s)=\frac{5.75 s^{3}-18.3 s^{2}+92.4 s}{s^{5}+8.33 s^{4}+33.0 s^{3}+74.8 s^{2}+94.5 s+52.3}
$$

Letting $\sigma_{1}=\sigma_{2}=1, \sigma_{3}=\sigma_{4}=1 / 3, \sigma_{5}=1 / 5$, the SFG realization of (7) using the methodology mentioned in section 3 is shown in Fig. 3.

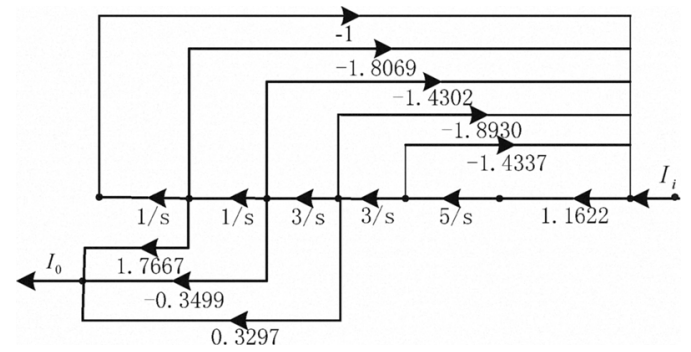

Fig.3. SFG realization of wavelet filter

To realize the integrators and branch coefficients in Fig.3, we extend the structure of the basic SI bilinear integrator in Fig.2 so that it includes these additional coefficients as shown in Fig.4. Fig.4(a) implements this multiple-input multipleoutput bilinear integrator, in which the negative output is 
achieved by simply inverting the output current of the integrator with a simple current mirror circuit. Fig.4(b) is the simplified form of Fig.4(a). The SI implementation of the first derivative of Gaussian wavelet filter presented in Fig. 3 can be obtained as shown in Fig.5.

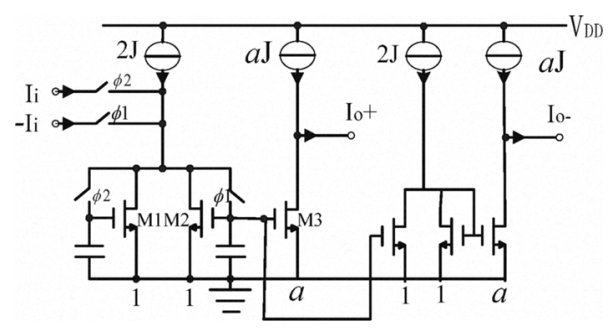

(a)

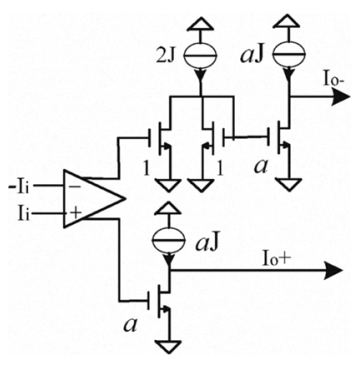

(b)

Fig.4. (a)Multiple-input multiple-output bilinear integrator and (b) Simplified form

One can denormalize (7) to any desired centre frequency according to application requirement. Herein, the centre frequency is selected as $5 \mathrm{KHz}$ as for the auditory wavelet transform. Meanwhile, the SI circuit is a sampled-data system in which the minimum sampling frequency can be determined by the sampling theorem. However, the greater the sampling frequency $f_{s}$, the smaller the W/L of the current mirror; in this case we let $f_{s}=50 \mathrm{KHz}$. In addition, to avoid errors due to the bilinear transform, frequency pre-warping is used, which can be expressed as:

$$
\omega_{p}=2 f_{s} \tan \frac{\omega_{0}}{2 f_{s}}
$$

where $\omega_{0}$ and $\omega_{\mathrm{p}}$ are the frequencies in the z-domain and sdomain, respectively.

From (4)-(8), the parameters for the wavelet filter can be determined which are listed in Table I.

\section{Simulation Results}

In this paper, we use the program ASIZ to simulate SI circuits [12]. ASIZ (Analysis of SI Filters in Z Transform) uses an interface with graphic windows, and analyses switched-current, switched-capacitor filters, or any periodically switched circuit where the circuit stabilizes between the switching instants.

With the sampling frequency $50 \mathrm{KHz}$, the time-domain response of the wavelet filter at $a=2^{0}$ is plotted in Fig.6. The peak value is achieved at around $\mathrm{t}=0.06 \mathrm{~ms}$, which is almost the same as the ideal value $0.056 \mathrm{~ms}$. As seen from Fig.6, the performance of the SI wavelet filter is confirmed by the excellent approximation of the first derivative of Gaussian wavelet.

Due to the feature of implementing wavelet transforms using SI filters, any dilation constant across different scales of the transform can be precisely implemented and easily controlled by the sampling frequency. Changing the sampling frequency to $100 \mathrm{KHz}$ and $200 \mathrm{KHz}$ for example, we can implement the first derivative of Gaussian wavelet filters at dyadic scale values $a=2^{-1}$ and $a=2^{-2}$, respectively. The timedomain responses are shown in Fig.7, achieving the peak value at $\mathrm{t}=0.03 \mathrm{~ms}$ and $\mathrm{t}=0.015 \mathrm{~ms}$, respectively. Observed from these figures, simulation results indicate that the presented approach can easily approximate an arbitrary wavelet function at different dilations by controlling the sampling frequency.

\section{Conclusions}

Hardware implementation is an optimum approach to the real-time application of wavelet transforms. This paper has described a novel method for the SI filter implementation of wavelet transform, in which the SFG methodology is utilized to implement the arbitrary approximation rational functions of wavelet bases. Taking the first derivative of Gaussian wavelet as an example, the proposed approach employs the bilinear mapping lossless integrators in the wavelet filter design. The simulation results have shown that the proposed method is very suitable for the analogue implementation of arbitrary wavelet bases.

TABLE I

PARAMETERS OF SI SFG WAVELET FILTER

\begin{tabular}{|c|l|l|l|l|l|l|l|l|l|l|l|l|}
\hline$\alpha_{1}$ & $\alpha_{2}$ & $\alpha_{3}$ & $\alpha_{4}$ & $\alpha_{5}$ & $\alpha_{6}$ & $\alpha_{7}$ & $\alpha_{8}$ & $\alpha_{9}$ & $\alpha_{10}$ & $\alpha_{11}$ & $\alpha_{12}$ & $\alpha_{13}$ \\
\hline 1.16 & 1.14 & -1.65 & 0.69 & -1.30 & 0.23 & 0.69 & -0.99 & -0.24 & 0.23 & -0.42 & 0.41 & 0.23 \\
\hline
\end{tabular}




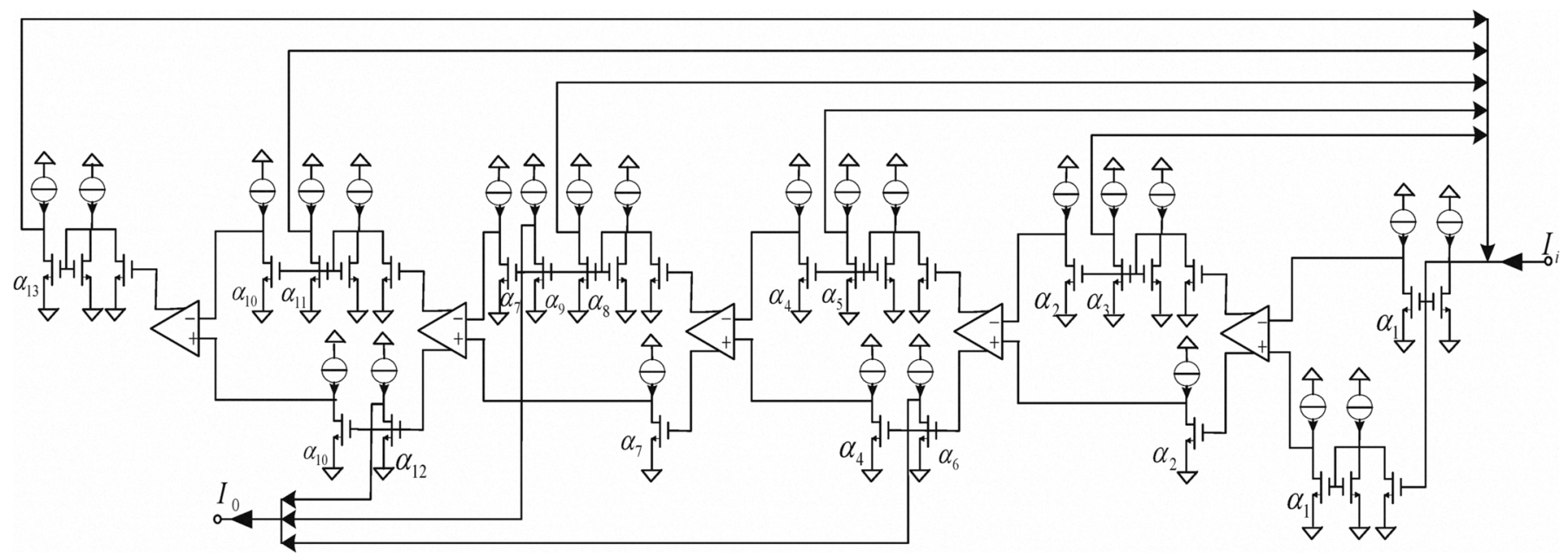

Fig.5. SI circuit of SFG wavelet filter

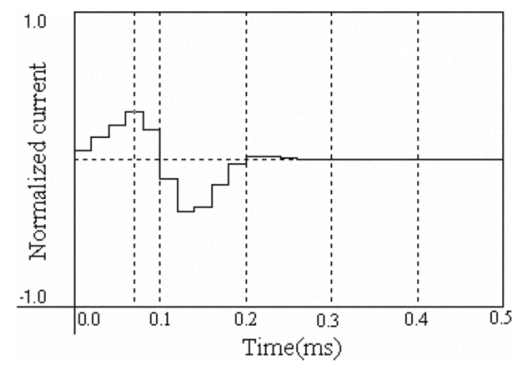

Fig.6. Time-domain response of the wavelet filter at $a=2^{0}$

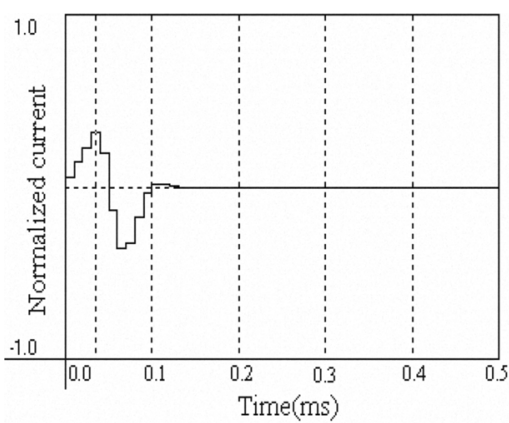

(a)

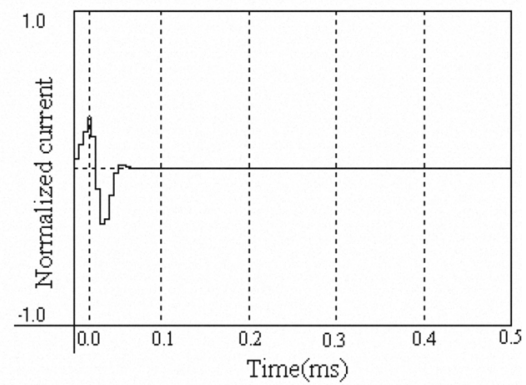

(b)

\section{REFERENCES}

[1] Mallat, S., A Wavelet Tour of Signal Processing. New York: Academic Press, 2001

[2] Tay, P.C., and Havlicek, J.P.: 'Frequency implementation of discrete wavelet transforms', IEEE Southwest Symp. Image Analysis and Interpretation, 2004, pp. 167-171

[3] Huang, C.T., Tseng, P.C., and Chen, L.G.: 'Analysis and VLSI architecture for 1-D and 2-D discrete wavelet transform', IEEE Trans. Signal Process., 2005, 53, pp. 1575-1586

[4] Lin, J., Ki, W.H., Edwards, T., and Shamma, S.: 'Analog VLSI implementations of auditory wavelet transforms using switched-capacitor circuits', IEEE Trans. Circuits and Syst., 1994, 41(9), pp. 572-583

[5] Haddad, S.A.P., Verwaal, N., Houben, R., and Serdijn, W.A.: 'Optimized dynamic translinear implementation of the Gaussian wavelet transform', ISCAS, 2004, pp. 145-148

[6] Haddad, S.A.P., Bagga, S., and Serdijn, W.A.: 'Log-domain wavelet bases', IEEE Trans. Circuits and Syst., 2005, 52(10), pp. 2023-2032

[7] Hu, Q.C., He, Y.G., Liu, M.R., Guo, D.X., and Li, H.M.: 'Design and implementation of wavelet filter using switched-current circuits', IEEE TENCON, 2005

[8] Zhao, W.S., Sun, Y., Huang, Q. X., Zhu, X., and He, Y.G.: 'Analogue VLSI realization of wavelet transforms for cochlear implant using switched-current circuits', Proc. ASP, 2008, pp. 8-13

[9] Sun, Y., Design of High Frequency Integrated Analogue Filters. Stevenage: IEE Press, 2002

[10] Sun, Y., and Fidler, J.K.: 'Some design methods of OTA-C and CCII-RC filters', IEE Colloquium on Digital and Analogue Filters and Filtering Systems, 1993, 2

[11] Toumazou, C., Hughes, J.B., and Battersby, N.C.: 'Switched-Currents: an Analogue Technique for Digital Technology' (Short Run Press, 1993)

[12] de Queiroz, A.C.M, Pinheiro, P.R.M., and Caloba, L.P.: 'Systematic nodal analysis of switched-current filters', IEEE Int. Symp. Circuits Syst., 1991, 3, pp. 1801-1804 